\title{
Rationale for statistical characteristics of road safety parameters
}

\author{
Tatiana Dormidontova ${ }^{1}$, and Sergey Evdokimov ${ }^{1, *}$ \\ ${ }^{1}$ Samara State Technical University, Institute of Architecture and Civil Engineering, \\ 194, Molodogvardeyskaya St., 443001, Samara, Russia
}

\begin{abstract}
When making engineering decisions at the stage of designing auto-roads and man-made structures it is necessary to take into account the statistical variability of physical and mechanical characteristics of the used materials as well as the different effects on the structures. Thus the rationale for the statistical characteristics of the parameters that determine the reliability of roads and man-made engineering facilities is of particular importance.There are many factors to be considered while designing roads, such as natural climatic factors, the accidental effects of the operating loads, the strength and deformation characteristics of the materials, the geometric parameters of the structure, etc. which affect the strength characteristics of roads and man-made structures. The rationale for statistical characteristics of the parameters can help an engineer assess the reliability of the decision and the economic risk, as well as avoid making mistakes in the design of roads and man-made structures.However, some statistical characteristics of the parameters that define the reliability of a road and man-made structures play a key role in the design. These are the visibility distance in daytime for the peak curve, variation coefficient of radial acceleration, the reliability of visibility distance and other parameters.
\end{abstract}

\section{Introduction}

Nowadays there exist a lot of problems in road and highway traffic management. This is due to the increase in the number of automobiles, historically established street-road network of cities as well as the parking lots on the driveway. All these factors decrease the traffic safety and the highway traffic capacity causing traffic jams [1-3].

Great improvements in city traffic can be achieved by taking urban planning measures such as bridge, tunnel and highway construction [4-5]. The development of such projects is expensive and time consuming.

The relevance of the technical solutions is based on a comparison of different techniques of traffic management and the ways of their technical implementation. It takes into account the specificities and characteristics of the designed sections of roads and the current domestic and foreign experience of operating similar systems.

*Corresponding author: adisk63@yandex.ru 


\section{Materials and Methods}

The paper considers statistical characteristics of parameters determining the reliability of roads and man-made structures in details.

Variation coefficient of curvature radius of vertical curve is so small that it is meaningless to evaluate it. For example, the average radius is $25 \mathrm{~km}$. The maximum possible deviation caused by the inaccuracy of pegging-out is $10 \mathrm{~m}$. Variation coefficient is $V=10 / 25000=1 / 2500=0.04 \%$. Its value is too small to be taken into account in calculations.

The visibility distance in daytime for the peak curve is obtained by the following:

$$
L=\left(\sqrt{h_{1}}+\sqrt{h_{2}}\right) \times \sqrt{2 R}
$$

where $h_{1}$ and $h_{2}$ are, respectively, the elevation of the driver above the road and the rise of the obstacle to be visible; $R$ - radius of the vertical curve.

The average visibility distance $\bar{L}$ and standard visibility distance $\sigma_{L}$ standard are calculated by the following formulas:

$$
\begin{aligned}
& \bar{L}=\left(\sqrt{\overline{h_{1}}}+\sqrt{\overline{h_{2}}}\right) \times \sqrt{2 \bar{R}}, \\
& \sigma_{L}=\frac{1}{2} \times \sqrt{2 R \times\left(\frac{\sigma_{h_{1}}^{2}}{h_{1}}+\frac{\sigma_{h_{2}}^{2}}{h_{2}}\right) .} .
\end{aligned}
$$

Calculated value of the visibility distance:

$$
L=\bar{L}+n \times \sigma_{L} .
$$

The average distance from the driver's eyes to the surface of the road is $120 \mathrm{~cm}$. The range of the driver's eyes distance from the road surface is $30 \mathrm{~cm}$. For this indicator the normal distribution is accepted [6,7]. Standard value for a driver's eye elevation above the road surface is $h_{1}=5 \mathrm{~cm}$. Variation coefficient is $V=0.166$.

The height of the road bump is taken as the obstruction on the road surface, $h_{2}=20 \mathrm{~cm}$. The height of the road bump is $15 \div 25 \mathrm{~cm}$. The default value, $h_{2}=1.6 \mathrm{~cm} V=0.16$.

Thus, when $R=70000 \mathrm{~m}, h_{1}=120 \mathrm{~cm}$ and $h_{2}=10 \mathrm{~cm}$

$$
\begin{aligned}
& L=(\sqrt{120}+\sqrt{10}) \times \sqrt{2 \times 7000000}=527.7 \mathrm{~m} \\
& \sigma_{L}=\frac{1}{2} \times \sqrt{2 \times 7000000 \times\left(\frac{25}{120}+\frac{2.56}{10}\right)}=12.7 \mathrm{~m} .
\end{aligned}
$$

Variation coefficient $V=0.024$.

For a concave curve, the car speed $v$ and the speed standard $\sigma$ under the conditions of overloading of the bumpy and the passenger's overall health are calculated by the formulas:

$$
V=\sqrt{R \times b}
$$




$$
\sigma=\frac{\partial v}{\partial b} \times \sigma_{b}=\frac{\sqrt{R}}{2 \times \sqrt{b}} \times \sigma_{b}=0.5 \sqrt{\frac{R}{b}} \times \sigma_{b},
$$

where, $b$-normative radial acceleration, that is $0.5 \div 0.7 \mathrm{~m} / \mathrm{s}^{2}$.

Variation coefficient is calculated by the formula:

$$
V=\frac{0.5 \times \sigma_{b} \times \sqrt{R}}{\sqrt{b} \times \sqrt{R} \times \sqrt{b}}=0.5 \frac{\sigma_{b}}{b}=0.5 v_{b} .
$$

Variation coefficient of radial acceleration can be determined by the following factors, i.e. the range of radial acceleration changes has 6 root-mean-square deviations [8-10]. Consequently, the standard is $0.2 / 6=0.03 \mathrm{~m} / \mathrm{s}^{2}$, while variation coefficient is $V_{b}=0.03 / 0.6=0.05$ or $5 \%$.

Thus, the average car speed value on the concave curve when $R=8000 \mathrm{~m}$ and the average value of $b=0.6 \mathrm{~m} / \mathrm{s}^{2}$ is $\bar{v}=\sqrt{8000 \times 0.6}=69.3 \mathrm{~m} / \mathrm{s}=41,6 \mathrm{~km} / \mathrm{h}$; root-mean-square deviations $\sigma=0.5 \times \sqrt{8000 / 0.6} \times 0.03=1.73 \mathrm{~m} / \mathrm{s}=0.104 \mathrm{~km} / \mathrm{h}$; variation coefficient of the traffic speed is $0.104 / 41.6=0.0025$.

The calculated visibility distance in darkness hours with headlamp lightning is calculated by the formula:

$$
L=\sqrt{2 R \times h_{F}}
$$

where, $h_{F}$ - elevation of the center of the headlamp above the road surface.

Average value, standard and the variation coefficient are calculated by the formulas:

$$
\begin{aligned}
& \bar{L}=\sqrt{2 \bar{R} \times \overline{h_{F}}}, \\
& \sigma_{L}=0.5 \times \sqrt{\frac{R}{h_{F}}} \times \sigma_{h_{F}}, \\
& V=0.5 \times V_{h_{F}} .
\end{aligned}
$$

The following values were analyzed as the parameters of the study: $R=8000 \mathrm{~m}$, $h_{F}=0.6 \mathrm{~m}, V_{h_{F}}=0.01 / 0.6$.

Then the calculated values are: $\bar{L}=\sqrt{2 \times 8000 \times 0.6}=98 \mathrm{~m}$, $\sigma_{L}=0.5 \times \sqrt{8000 / 0.6} \times 0.01=1.15 \mathrm{~m}$ and $V=0.5 \times(0.01 / 0.6)=0.017$.

Thus, variation coefficient values of vertical curve parameters are negligible:

- for the visibility distance in daytime for the peak curve $V=0.024$,

- for car speed on a concave curve $V=0.0025$,

- for visibility distance in darkness hours with headlamp lightning on a concave curve $V=0.017$.

When determining the reliability of visibility distance normative values for visibility distance were used.

For Category II road with the traffic speed of $120 \mathrm{~km} / \mathrm{h}$ the visibility distance is $250 \mathrm{~m}$, $V$ is $20 \%$, thus $\sigma_{V}=V \times \bar{L}=250 \times 0.2=50 \mathrm{~km} / \mathrm{h}$.

Then the reliability is calculated in the following way: 


$$
P=f(Z)=f\left(\frac{\bar{L}-L_{n}}{\sqrt{\sigma_{L}^{2}+\sigma_{n}^{2}}}\right)=f\left(\frac{250-98}{\sqrt{1.15^{2}+50^{2}}}\right)=f(3.03)=0.998
$$

The average value of a road visibility distance is evaluated like this:

$$
\bar{S}=\frac{\bar{v}}{3.6}+\frac{\bar{k} \times \bar{v}}{254(\bar{\varphi} \pm i+\bar{f})}+L_{b} .
$$

The standard is calculated by the formula:

$$
\sigma=\sqrt{\left(\begin{array}{l}
\left.\left(\frac{1}{3,6}+\frac{2 \times v \times k}{254(\varphi \pm i+f)}\right) \times \sigma_{v}\right)^{2}+\left(\frac{v^{2}}{254(\varphi \pm i+f)} \times \sigma_{k}\right)^{2}+ \\
+\left(\frac{k \times v^{2}}{254(\varphi \pm i+f)^{2}} \times \sigma_{\varphi}\right)^{2}+\left(\frac{k \times v^{2}}{254(\varphi \pm i+f)^{2}} \times \sigma_{f}\right)^{2}
\end{array}\right.}
$$

where, $v$ - car speed at the start of the brake; $k$ - braking performance coefficient (minimum value 1.2 , maximum 2.3 , average value 1.75 , standard 0.18 , variation coefficient $0.1) ; \varphi$ - coefficient of adhesion $(\varphi=0.2) ; f$ - tractive resistance $(f=0.35) ; i$ - longitudinal slope of the road; $L_{b}$ - security clearance.

\section{Results}

On the basis of the obtained data the section of the road can be calculated by the formulas given above and the following results can be got, when $v=60 \mathrm{~km} / \mathrm{h}, \bar{S}=152.4 \mathrm{~m}$, $\sigma=8.04 \mathrm{~m}, V=0.05$.

The formula for calculating the storm water can be written as follows:

$$
Q_{L}=16.7 \times a_{r} \times F \times \alpha \times \varphi,
$$

Where, $a_{r}-$ the calculated intensity of the downpour of the same probability of exceedance as the amount of the desired $\mathrm{mm} / \mathrm{min} ; F-$ drainage area; $\alpha$ - run-off coefficient; $\varphi$ - reduction coefficient (imperfection of run-off), thus:

$$
\begin{gathered}
\varphi=\frac{1}{\sqrt[4]{10 F}}, \\
a_{r}=\left(\frac{60 \times v_{d}}{L}\right)^{2 / 3} \times a_{t}=K_{T} \times a_{t} .
\end{gathered}
$$

Where, $a_{t}-$ the average intensity of one-hour heavy rain, $\mathrm{mm} / \mathrm{min} ; L-$ basin length, $\mathrm{km} ; v_{d}$ - water velocity, $\mathrm{km} / \mathrm{min} ; K_{T}-$ transition code.

Then, the formula (17) looks like this:

$$
Q_{L}=16.7 \times a_{t} \times K_{T} \times F \times \varphi .
$$

Taking into account the road area and the natural slope area the following formula can be derived: 


$$
Q_{L}=16.7 a_{t}\left(5.24 \times F_{d}+K_{T} \frac{F_{o}}{\sqrt[4]{10 F_{o}}}\right),
$$

Where, $F_{d}$ - road area; $F_{o}$ - natural slope area.

On the basis of the got results a graph can be made showing the dependency of reliability $P$ on the average intensity of one-hour heavy rain $a_{t}$, Fig.1.

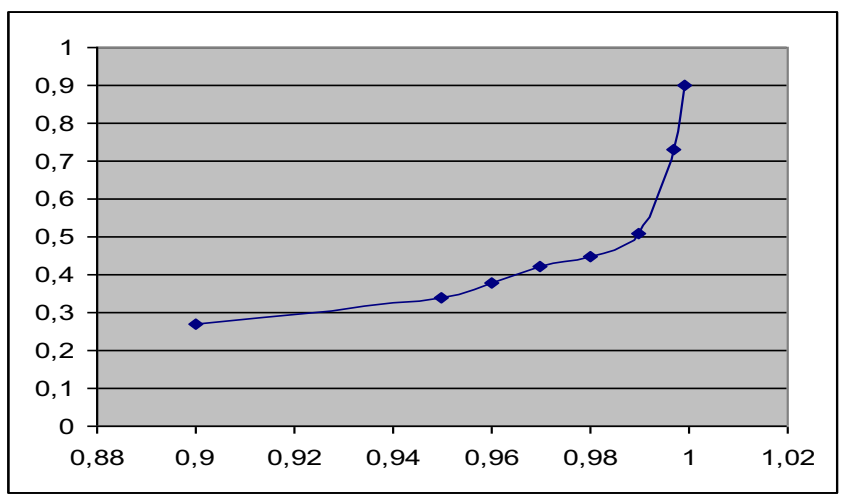

Fig.1. The dependency of reliability on the average intensity of one- hour heavy rain.

The values of $\overline{a_{t}}$ with the probability of $1,2,3$, and $4 \%$ (these values are accepted for calculation depending on the road category) are of interest [11].

$\sigma_{a_{t}}$ and $V_{a_{t}}$ for all of four probability values have been calculated and the results are given in Table1.

Table 1. Calculated values $\sigma_{a_{t}}$ and $V_{a_{t}}$ depending on probability.

\begin{tabular}{|c|c|c|c|c|c|c|c|}
\hline \multicolumn{2}{|c|}{$1 \%\left(a_{t}=0.59\right)$} & \multicolumn{2}{c|}{$2 \%\left(a_{t}=0.465\right)$} & \multicolumn{2}{c|}{$3 \%\left(a_{t}=0.42\right)$} & \multicolumn{2}{c|}{$4 \%\left(a_{t}=0.38\right)$} \\
\hline$\sigma_{a_{t}}$ & $V_{a_{t}}$ & $\sigma_{a_{t}}$ & $V_{a_{t}}$ & $\sigma_{a_{t}}$ & $V_{a_{t}}$ & $\sigma_{a_{t}}$ & $V_{a_{t}}$ \\
\hline $\begin{array}{c}0.093 \\
\mathrm{~mm} / \mathrm{min}\end{array}$ & $16 \%$ & $\begin{array}{c}0.03 \\
\mathrm{~mm} / \mathrm{min}\end{array}$ & $6.4 \%$ & $\begin{array}{c}0.023 \\
\mathrm{~mm} / \mathrm{min}\end{array}$ & $5.5 \%$ & $\begin{array}{c}0.013 \\
\mathrm{~mm} / \mathrm{min}\end{array}$ & $5.2 \%$ \\
\hline
\end{tabular}

\section{Discussion}

Therefore, the variation coefficient for road area tends to zero, because the error in its measurements is negligible. In order to assess the reliability of the road section, it is necessary to have statistical characteristics of all parameters and determine the reliability of an auto-road in accordance with the known dependences of the reliability theory [12-15]. The algorithms for calculating the statistical characteristics of the parameters that determine the reliability of the road elements are reduced to the use of linearization and brute force methods taking into account the Monte Carlo method [16,17].

The research results are proved by checking all the results for homogeneity and determining the variation coefficient. It has been observed that in the vast majority of cases, individual structures and roads are operated without meeting the requirements. It results in the increased number of defects and rapid decrease of reliability to normalized values. The operation of items with the reliability of below normalized values does not guarantee their safety and efficiency. 


\section{Conclusions}

Nowadays the problem of estimating the statistical characteristics of road parameters is quite evident. The road parameters determined by analytical dependences are highly volatile because of the inaccuracy of the defining factors. However, every parameter of physical origin is statistically unstable. It is shown by the variation coefficient of their values. This causes the variability in the parameters that determine the reliability of a road. Their study may result in the changes of general and particular road design rules in order to increase their reliability. The methodology for estimating the statistical parameters that define the operating characteristics of a road has been determined. The statistical characteristics of road parameters have been estimated by the obtained statistical distributions. Statistical information on the material properties, geometric dimensions of the road elements and their statistical distributions acted as the initial data. On their basis the distribution of the analyzed parameter has been made by using normalized dependences.

\section{References}

1. Yu.M. Galitskova, Tradition and Innovation in Building and Architecture, 222-223 (2000)

2. L.G. Goverdovskaya, Urban Construction and Architecture 1, 72-80 (2015) (doi:10.17673/Vestnik.2015.01.12)

3. A.N. Davydov, Tradition and Innovation in Building and Architecture, 162-165 (2015)

4. T.V Dormidontova, Monograph, Sibak, 152 (2015)

5. T.V. Dormidontova, Patent to Useful Model 160415 (2016)

6. S.V. Evdokimov, Scientific Survey 22, 102-107 (2015)

7. B.G. Ivanov, Again on the roads of Russia, 112-117 (2015)

8. B.G. Ivanov, Tradition and Innovation in Building and Architecture, 36-37 (2013)

9. B.G. Ivanov, Tradition and Innovation in Building and Architecture, 47 (2013)

10. E.I. Marinin, Urban Construction and Architecture 4, 80-84 (2014) (doi:10.17673/Vestnik.2014.04.12)

11. A.A. Mikhasek, Prirodoohranyie I gidrotehnicheskie sooruzheniya, 398-401 (2014)

12. V.A. Pavlova, Ways of Improving the Quality of Roads, 158-168 (2015)

13. L.V. Pavlova, Urban Construction and Architecture 2, 96-103 (2016) (doi:10.17673/Vestnik.2016.02.18)

14. L.V. Pavlova, Ways of Improving the Quality of Roads, 168-176 (2015)

15. S.A. Piyavskiy, Vestnik MGSU, 54-61 (2012)

16. M.V. Rodionov, Geotec Hanoi, 399-402 (2013)

17. A.V. Filatova Ways of Improving the Quality of Roads, 206-211 (2015) 